\title{
Dificuldades diárias associadas às próteses totais
}

\author{
Daily difficulties associated with full conventional dentures
}

Flávia Christiane de Azevedo Machado ${ }^{1}$

Anna Paula Serêjo da Costa ${ }^{2}$

Anna Lepríncia Bezerra Pontes ${ }^{2}$

Kenio Costa Lima ${ }^{2}$

Maria Ângela Fernandes Ferreira ${ }^{2}$

${ }^{1}$ Instituto Federal de Educação, Ciência e

Tecnologia do Rio Grande do Norte. Av. Senador Salgado Filho 1559, Tirol. 59.015-000 Natal RN.

flavitamachado@yahoo.com.b

${ }^{2}$ Universidade Federal do

Rio Grande do Norte.

\begin{abstract}
The effectiveness of health services can be evaluated from the quality of life (QOL) standpoint. Thus, this study evaluated rehabilitation services using full conventional dentures (FCD) of Specialized Dental Care Centers (SDCC) in Rio Grande do Norte (RN) regarding daily difficulties associated with these dentures made between 2007 and 2009. A cross-sectional study was conducted with 138 users of these FCD, collecting data by clinical examination and a questionnaire based on the Oral Impacts on Daily Performances index. The Fisher and chi-square tests were used to test the association between the variables. The result was that $42 \%$ of users reported difficulties in executing oral activities due to FCDs. These difficulties were more frequent and intense in the activities of eating, speaking and smiling. In general, $58.7 \%$ of users did not have functional teeth. In relation to the clinical evaluation of FCDs, $57.2 \%$ of upper and $9.2 \%$ of lower FCDs were satisfactory. There was an association between difficulty and the absence of functional teeth, but not with inadequate FCDs. Thus, the SDCCs were effective in upper FCD rehabilitation, since the difficulties encountered are within the standard limitations of this type of rehabilitation. On the other hand, the cost-benefit of rehabilitation of lower FCDs must be evaluated.
\end{abstract}

Key words Oral health, Quality of life, Dentures
Resumo A efetividade de serviços de saúde pode ser avaliada pela ótica da qualidade de vida. Assim, este estudo avaliou o serviço de reabilitação a partir das dificuldades diárias associadas a próteses totais convencionais (PTC), produzidas entre 2007 e 2009, pelos Centros de Especialidades Odontológicas (CEO) de uma mesorregião do Rio Grande do Norte (RN). Realizou-se um estudo seccional com 138 usuários dessas PTC, coletando-se os dados por exame clínico e questionário baseado no indicador Oral Impacts on Daily Performances. Utilizaram-se os testes de Fisher e Qui-quadrado para testar associação das variáveis. Como resultado, $42 \%$ da amostra relatou dificuldades, relacionadas à PTC, para executar atividades bucais. Tais dificuldades foram mais frequentes e intensas nas atividades de comer, falar e sorrir. No total, $58,7 \%$ da amostra não tinha dentes funcionais. Quanto à avaliação clínica das PTC, $57,2 \%$ das superiores e 9,2\% das inferiores estavam satisfatórias. Houve associação da presença de dificuldades e a ausência de dentes funcionais, mas não com PTC inadequadas. Assim, os CEO foram efetivos na reabilitação com PTC superior, já que as dificuldades encontradas integram limitações próprias a tal reabilitação. Por outro lado, o custo benefício da reabilitação inferior deve ser reavaliado.

Palavras-chave Saúde bucal, Qualidade de vida, Prótese dentária 


\section{Introdução}

As políticas de Promoção da Saúde devem atuar sobre os fatores relacionados à qualidade e vida e bem-estar da população para serem efetivas ${ }^{1}$.

$\mathrm{O}$ conceito de qualidade de vida $(\mathrm{QV})$ está associado à percepção de como as enfermidades, disfunções ou incapacidades interferem nos domínios físico, psicológico e social da vida de um indivíduo ${ }^{2}$. Contudo, a QV transcende a subjetividade, envolvendo parâmetros materiais mensuráveis e comparáveis, apesar da necessidade permanente de relativizá-los culturalmente no tempo e no espaço ${ }^{3}$.

Assim, a qualidade de vida tem sido utilizada como indicador de eficácia e eficiência das políticas públicas ${ }^{2}$. Todavia, seu uso ocorre em pequena escala por falta de informações das equipes de saúde de como investigá-la ${ }^{2}$.

No campo da saúde bucal, esta investigação pode ser feita pelos indicadores sociodentais como o Oral Impacts on Daily Performances (OIDP) ${ }^{4}$, já validado no Brasil ${ }^{5-8}$. O OIDP descreve se houve dificuldade na realização de atividades relativas à boca associadas ao domínio físico, psicológico e social, bem como a causa, frequência e severidade desta dificuldade ${ }^{4}$.

$O$ uso destes indicadores para estimar a efetividade de intervenções odontológicas condiz com o referencial teórico da bucalidade ${ }^{9,10}$. Tal referencial preconiza a requalificação da assistência odontológica, eminentemente técnica, para uma na qual não só a materialidade como também as funções sociais da boca e suas estruturas sejam consideradas no planejamento, prognóstico e avaliação das intervenções em prol de sua resolutividade ${ }^{11}$.

No Brasil, o edentulismo foi priorizado como uma questão de saúde pública pela política brasileira de saúde bucal que instituiu os Centros de Especialidades Odontológicas $(\mathrm{CEO})^{12}$. Estes Centros devem, dentre outras atribuições, realizar reabilitações protéticas para reduzir o perfil de morbidade da perda dentária.

O edentulismo inicia-se precocemente, entre os 15 e 19 anos de idade ${ }^{13}$, prosseguindo até a senescência. Recentemente, o levantamento epidemiológico SB Brasil 2010 evidenciou que mais de 3 milhões de idosos precisam de prótese total convencional bimaxilar ${ }^{14}$.

No entanto, a reabilitação protética necessita ser avaliada quanto à sua efetividade no dia-adia dos usuários por influenciar a percepção de qualidade de vida destes ${ }^{15}$. Segundo Redford et al. ${ }^{15}$, os usuários de prótese total convencional
(PTC) relatam dificuldades para mastigar, diminuição da autoestima e do contato social devido aos problemas de instabilidade das próteses mandibulares.

Em virtude da relevância social dos CEO, é importante avaliar o produto de suas ações para assegurar o direito a uma assistência resoluti$\mathrm{va}^{16}$. Com este intuito, o presente estudo utilizou a qualidade de vida, na perspectiva das dificuldades diárias, como indicador de efetividade das reabilitações com próteses totais convencionais (PTC) realizadas em CEO da mesorregião Leste Potiguar do RN entre 2007 e 2009.

\section{Método}

Este é um estudo transversal conduzido nos CEO da mesorregião Leste Potiguar do Rio Grande do Norte que realizam reabilitação com PTC há ao menos 1 ano $^{17}$. Essa é a região com maior potencial socioeconômico do $\mathrm{RN}$ e, quanto ao cenário público odontológico, comporta CEO com destacada qualidade no âmbito nacional segundo o prêmio Brasil Sorridente do Conselho Federal de Odontologia ${ }^{18}$.

O presente estudo envolveu uma amostra de 150 indivíduos reabilitados com PTC há no mínimo 1 ano nos CEO dos municípios de Natal, Parnamirim, São Gonçalo do Amarante e Macaíba.

O tamanho desta amostra, selecionada intencionalmente, foi baseado em estudo piloto com o objetivo de aferir a prevalência de dificuldades relacionadas às PTC confeccionadas entre $2007 \mathrm{e}$ 2009 nos CEO sob estudo. Deste piloto participaram 30 indivíduos captados entre os $1882 \mathrm{com}$ registro da referida reabilitação nos livros de atendimentos dos CEO e que tinham informações sobre nome, telefone e/ou endereço atualizados. Desta forma, obteve-se $45 \%$ de prevalência de dificuldades que aliada aos parâmetros de margem de erro $(20 \%)$ e taxa de não resposta $(20 \%)$ permitiram estimar a amostra em 141 indivíduos. Portanto, a quantidade de pessoas captadas ( $\mathrm{n}=$ 150) foi superior ao valor obtido com os dados de estudo piloto realizado $(\mathrm{n}=141)$.

Todos os participantes do estudo foram informados sobre os objetivos e o protocolo da pesquisa, previamente aprovados pelo comitê de ética da Universidade Federal do Rio Grande do Norte.

O protocolo da pesquisa envolvia submissão a exame clínico e ao questionário baseado no indicador sociodental Oral Impacts on Daily Performances (OIDP-CS) ${ }^{19}$. O exame clínico buscava estimar 
a quantidade de dentes perdidos e a presença de dentes funcionais dos participantes do estudo, bem como a qualidade técnica de suas PTC.

\section{Exame clínico}

O exame clínico foi realizado sob luz natural e artificial através de sonda OMS e espelho plano com cabo. Tal exame visava verificar a quantidade de dentes perdidos e se havia dentes funcionais, ou seja, dentes sem indicação para exodontia conforme critérios utilizados pelo levantamento epidemiológico brasileiro SB Brasil 2010 (A cárie destruiu o dente de tal modo que não é possível restaurá-lo; a doença periodontal progrediu tanto que o dente está com mobilidade e, no julgamento clínico do examinador, não pode ser recuperado por tratamento periodontal $)^{16}$.

O exame técnico da prótese para classificá-la como satisfatória ou não, realizado por dentista treinado, também foi baseado no protocolo do SB Brasil $2010^{16}$. Seguindo este protocolo, as próteses foram examinadas quanto à retenção (avaliada se estava folgada ou apertada); estabilidade e reciprocidade (avaliada se havia deslocamento ou movimento de báscula); estética (avaliada se havia manchas ou fraturas e se não estava adequada ao perfil facial do paciente) e fixação (avaliada se havia lesões potencialmente decorrentes do uso). O critério de fixação não considerou a presença de candidose quando relacionada ao hábito de higiene do indivíduo. Ao final, a PTC foi classificada como insatisfatória caso algum critério estivesse inadequado ${ }^{20}$.

\section{OIDP-CS}

O OIDP-CS ${ }^{19}$ captou a existência ou não de dificuldades, nos últimos 6 meses, para comer, falar, higienizar os dentes, dormir, sorrir, manter equilíbrio emocional, trabalhar/estudar e conviver socialmente relacionadas ao uso das PTC confeccionadas nos CEO. Além disso, verificou a frequência, o grau da dificuldade (severidade), o principal sintoma (dor, desconforto, limitação funcional, estética e insatisfação com a aparência) e a causa percebidos pelo indivíduo como responsáveis pela dificuldade. Opções de causa foram disponibilizadas para que uma fosse escolhida (úlcera bucal/afta, pontos doloridos na boca, prótese desconfortável, com adaptação deficiente, com retenção deficiente, com cor dos dentes insatisfatória, com posicionamento dos dentes insatisfatório, prótese retém alimento). Somente se o indivíduo relatasse alguma causa relacionada à PTC é que seria considerada a existência de dificuldade.

A frequência e a severidade poderiam variar do escore 0 ( sem dificuldade) ao $5^{4,19}$. O produto do escore da frequência e severidade forneceu a intensidade da dificuldade que poderia variar de 0 a 25 (valor 20 ou 25: intensidade muito severa; 12, 15 ou 16: severa; 8,9 ou 10: moderada; 5 ou 6 : pouca; e, 1, 2, 3 ou 4: muito pouca $\left.{ }^{21}\right)$. Ainda foi calculada a extensão das dificuldades, ou seja, o número de atividades com execução dificultada em função das PTC. Desta forma, a extensão poderia variar do 0 a 8 .

\section{Análise dos dados}

As variáveis categóricas foram descritas quanto às frequências absoluta e relativa, enquanto as quantitativas por sua média, mediana, valores máximo/mínimo e desvio-padrão. As variáveis quantitativas foram categorizadas com base em sua distribuição e nas medidas de tendência central para possibilitar a análise bivariada pelos testes Qui-quadrado e Exato de Fisher com significância de $5 \%$.

\section{Resultados}

Em uma amostra de 150 indivíduos, houve uma perda de $8 \%(\mathrm{n}=12)$, totalizando 138 pessoas avaliadas. As causas das perdas foram: óbito, recusa, doença cognitiva incapacitante e desuso das PTC.

A maioria dos 138 participantes era do sexo feminino $(74,6 \%)$, casada $(63 \%)$, com idade média de 59,3 anos $( \pm 11,34)$, apresentava renda de 1 salário mínimo (44,2\%) e grau de escolaridade baixa (média de 5,13 anos de estudo $\pm 3,89$ ). Dos 138 participantes, $44,2 \%$ foram reabilitados com PTC superior e 55,8\% com PTC superior e inferior nos $\mathrm{CEO}$ sob estudo.

Em relação ao histórico de edentulismo, 82,6 $\%$ das pessoas já usavam PTC superior e 42,75\% PTC inferior antes da reabilitação nos CEO. Já o tempo médio de uso foi de 20,12 ( $\pm 13,57)$ e 10,51 $( \pm 13,83)$ anos, respectivamente, para próteses superiores e inferiores.

O exame clínico da boca revelou que as pessoas haviam perdido em média 29,16 ( $\pm 3,62$ ) dentes, contudo, em 58,7\% dos indivíduos havia presença de dentes funcionais.

No tocante à qualidade técnica das próteses, $57,2 \%$ das PTC superiores e 9,2\% das inferiores estavam satisfatórias. 
Em relação à qualidade de vida associada à saúde bucal, 58\% dos usuários não relataram dificuldades na execução das atividades englobadas no OIDP-CS. Além disso, dos indivíduos que relataram alguma dificuldade, esta aconteceu em apenas uma das atividades aferidas pelo OIDPCS (média $=0,99 \pm 1,58$; Mín: 0, Máx: 6).

Dentre as atividades impactadas, comer, falar e sorrir concentraram as maiores frequência e grau de severidade de dificuldade (Gráfico 1). Estas atividades eram percebidas, sobretudo, pela limitação da função da PTC relacionada à retenção deficiente (Tabela 1 e Gráfico 2).

Não foi encontrada associação entre relato de dificuldades e inadequação técnica das próteses. Contudo, houve associação entre dificuldades e ausência de dentes funcionais (ver Tabela 2).

\section{Discussão}

O patamar material mínimo e universal para se falar em qualidade de vida diz respeito à satisfação das necessidades mais elementares da vida humana: alimentação, acesso à água potável, habitação, trabalho, educação, saúde e lazer. Ou seja, elementos materiais que têm como referência noções relativas de conforto, bem-estar e realização individual e coletiva ${ }^{3}$.

$\mathrm{Na}$ área da saúde, há duas tendências na conceituação do termo qualidade de vida: a mais genérica e a relacionada à saúde ${ }^{2}$. A primeira apresenta uma acepção mais ampla, cujos estudos não se restringem a indivíduos com agravos específicos, já a segunda está mais direcionada aos aspectos associados às enfermidades ou às intervenções em saúde².

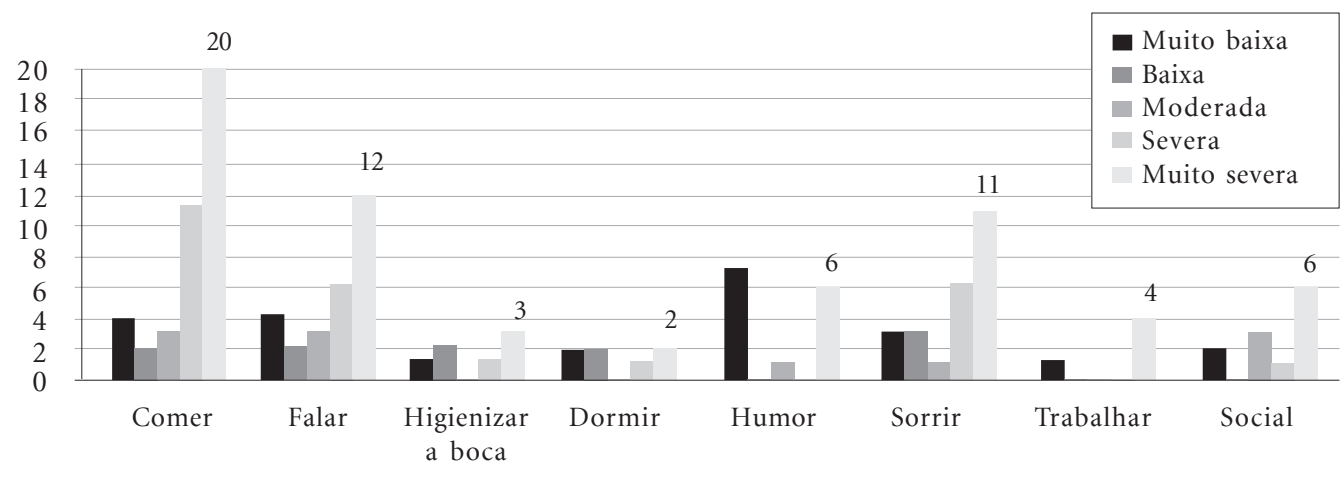

Gráfico 1. Frequência absoluta de indivíduos de acordo com a intensidade das dificuldades relacionadas ao uso das PTC confeccionadas nos CEO da Grande Natal entre 2007-2009.

Tabela 1. Número e frequência absoluta dos sintomas associados ao relato de dificuldades de acordo com as atividades avaliadas pelo OIDP-CS.

\begin{tabular}{|c|c|c|c|c|c|c|c|c|}
\hline \multirow{2}{*}{ Atividades } & \multicolumn{2}{|c|}{ Dor } & \multicolumn{2}{|c|}{ Desconforto } & \multicolumn{2}{|c|}{ Limitação funcional } & \multicolumn{2}{|c|}{ Insatisfação com a aparência } \\
\hline & $\mathbf{N}$ & $\%$ & $\mathbf{N}$ & $\%$ & $\mathbf{N}$ & $\%$ & $\mathbf{N}$ & $\%$ \\
\hline Comer & 16 & $11,6 \%$ & 6 & $4,3 \%$ & 32 & $24 \%$ & 0 & 0 \\
\hline Falar & 6 & $4,3 \%$ & 9 & $6,5 \%$ & 21 & $15,2 \%$ & 3 & $2,2 \%$ \\
\hline Higiene & 6 & $4,3 \%$ & 3 & $2,2 \%$ & 0 & 0 & 6 & $4,3 \%$ \\
\hline Dormir & 7 & $5,1 \%$ & 6 & $4,34 \%$ & 2 & $1,4 \%$ & 0 & 0 \\
\hline Humor & 6 & $4,3 \%$ & 3 & $2,2 \%$ & 9 & $6,5 \%$ & 3 & $2,2 \%$ \\
\hline Sorrir & 0 & 0 & 6 & $4,34 \%$ & 14 & $10,1 \%$ & 14 & $10,1 \%$ \\
\hline Trabalhar & 2 & $1,4 \%$ & 2 & $1,4 \%$ & 3 & $2,2 \%$ & 2 & $1,4 \%$ \\
\hline Social & 0 & 0 & 6 & $4,34 \%$ & 5 & $3,6 \%$ & 7 & $5,1 \%$ \\
\hline
\end{tabular}




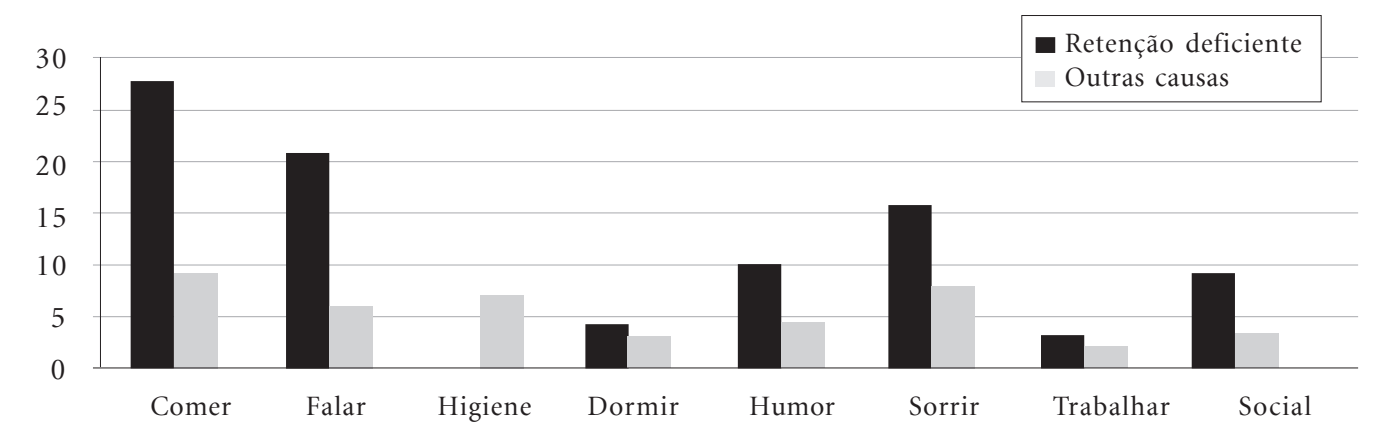

Gráfico 2. Frequência absoluta das causas apontadas como responsáveis pelas dificuldades associadas às PTC feitas nos CEO da Grande Natal entre 2007 e 2009.

Alguns autores defendem o enfoque mais específico, enquanto outros ressaltam que algumas medidas de qualidade de vida relacionada à saúde contribuem pouco para uma visão abrangente dos aspectos não médicos ${ }^{22}$. Contudo, os instrumentos de mensuração da qualidade de vida relacionada à saúde tendem a manter o seu caráter multidimensional ${ }^{2}$.

A qualidade de vida associada à saúde bucal (QVSB) está relacionada ao modo como a condição da boca afeta o dia-a-dia dos indivíduos ${ }^{23}$. Assim, a perda dentária influencia a QVSB, já que reduz a capacidade das pessoas de mastigar, falar e de se relacionar consigo e com o mundo ${ }^{24-27}$. Portanto, a reabilitação dessa condição deve permitir uma QVSB satisfatória no tocante à consecução de atividades próprias à boca. Nessa perspectiva, a reabilitação com PTC confeccionadas nos CEO foi positiva. Apesar de 42\% dos usuários relatarem dificuldades, estas ocorreram, na maioria dos indivíduos, em apenas uma das atividades realizadas, sendo considerada de baixa extensão. Essa frequência era esperada, já que esses indivíduos haviam perdido ao menos 20 dentes $^{28-30}$.

As dificuldades concentraram-se principalmente no domínio físico (1-comer, 2-falar), e psicológico (3-sorrir), sendo atribuídas, sobretudo, à retenção deficiente das PTC. A retenção deficiente é bastante relatada na literatura, principalmente nas próteses inferiores, como causa de desconforto, problemas com a mastigação, a aparência e a fala ${ }^{29-31}$.

No presente estudo, a limitação funcional das PTC foi o principal sintoma percebido para o relato de dificuldade. Heydeck et al. ${ }^{31}$ já evidenci- aram esse fato, relatando que os usuários de PTC relatavam apreensão quanto à perda de suas próteses ao beijar e durante o ato sexual em si. Segundo Critchlow e Ellis ${ }^{32}$ a altura deficiente de rebordo alveolar, sobretudo mandibular, no momento da reabilitação, estaria, provavelmente, associada à limitação funcional das PTC.

De fato, esse achado da limitação funcional não causou surpresa na medida em que, clinicamente, foi verificado um alto contingente de PTC tecnicamente insatisfatórias, sobretudo as inferiores. Contudo, não houve associação entre a frequência de dificuldades devido às PTC e a porcentagem de próteses insatisfatórias. Essa contradição já havia sido observada em estudos anteriores $^{33-35}$. Acredita-se que a ausência de associação deve-se a aspectos culturais e ao histórico do edentulismo.

O edentulismo é aceito em diversas culturas, sobretudo nas de países em desenvolvimento, como algo natural ao ciclo da vida ${ }^{36,37}$. Mas, na verdade, é um reflexo da falta de políticas de saúde para a manutenção dos dentes até idades mais avançadas ${ }^{38,39}$. Além disso, essa condição é mais comum entre pessoas de baixo poder aquisitivo, que tendem a se contentar com a reabilitação em si, independente de sua qualidade técnica ${ }^{40}$.

Quanto ao histórico do edentulismo, destacamos dois aspectos: o tempo e a capacidade de adaptação. A maioria das pessoas tem uma precoce história de perda dentária, estando no último nível do prejuízo funcional (perda dentária total) e seu referencial comparativo são próteses anteriores desgastadas. Dessa forma, tendem a se satisfazer com a reabilitação em si e se adap- 
Tabela 2. Valores absolutos, percentuais e significância estatística da associação entre frequência de dificuldades relacionadas a problemas com as PTC e condição sociodemográfica, histórico de uso anterior de prótese, histórico das PTC e condições de saúde bucal.

\begin{tabular}{|c|c|c|c|c|c|}
\hline \multirow{2}{*}{ Variável } & \multirow{2}{*}{ Categorias } & \multicolumn{2}{|c|}{$\begin{array}{l}\text { Dificuldade devido às PTC } \\
\text { confeccionadas nos CEO }\end{array}$} & \multirow[b]{2}{*}{$\begin{array}{l}\text { OR IC (95\%) } \\
\text { mín.-máx. }\end{array}$} & \multirow[b]{2}{*}{$\mathbf{P}$} \\
\hline & & $\underset{\mathbf{n}(\%)}{\operatorname{Sim}}$ & $\begin{array}{l}\text { Não } \\
\text { n }(\%)\end{array}$ & & \\
\hline \multirow[t]{2}{*}{ Idade $(\mathrm{N}=138)$} & Até 59 anos & $27(36)$ & $48(64)$ & 0,581 & 0,117 \\
\hline & Acima de 59 anos & $31(49,2)$ & $32(50,8)$ & $0,29-1,15$ & \\
\hline \multirow[t]{2}{*}{ Gênero $(\mathrm{N}=138)$} & Masculino & $18(51,4)$ & $17(48,6)$ & 1,668 & 0,192 \\
\hline & Feminino & $40(38,8)$ & $63(61,2)$ & $0,77-3,61$ & \\
\hline \multirow{2}{*}{$\begin{array}{l}\text { Situação conjugal atual } \\
(\mathrm{N}=138)\end{array}$} & Sem companhia & $19(37,3)$ & $32(62,7)$ & 0,731 & 0,384 \\
\hline & Com companhia & $39(44,8)$ & $48(55,2)$ & $0,36-1,48$ & \\
\hline \multirow[t]{2}{*}{ Escolaridade $(\mathrm{N}=138)$} & Até 04 anos de estudo & $27(37,5)$ & $45(62,5)$ & 0,677 & 0,26 \\
\hline & Acima de 04 anos de estudo & $31(47)$ & $35(53)$ & $0,34-1,34$ & \\
\hline \multirow[t]{2}{*}{ Renda mensal (N=138) } & Até $\mathrm{R} \$ 510,00$ & $42(40,8)$ & $61(59,2)$ & 0,818 & 0,61 \\
\hline & Acima de $\mathrm{R} \$ 510,00$ & $16(45,7)$ & $19(54,3)$ & $0,38-1,77$ & \\
\hline \multirow{2}{*}{$\begin{array}{l}\text { Uso anterior de PS } \\
(\mathrm{N}=138)\end{array}$} & Sim & $59(41,2)$ & $70(58,8)$ & 0,778 & 0,61 \\
\hline & Não & $9(47,4)$ & $10(52,6)$ & $0,29-2,06$ & \\
\hline \multirow{2}{*}{$\begin{array}{l}\text { Tipo anterior de } \mathrm{PS}^{*} \\
(\mathrm{~N}=119)\end{array}$} & PTC & $48(42,1)$ & $66(57,9)$ & 2,909 & 0,65 \\
\hline & PPR & $1(20)$ & $4(80)$ & $0,32-26,86$ & \\
\hline \multirow{2}{*}{$\begin{array}{l}\text { Tempo de uso da PS } \\
\text { anterior }(\mathrm{N}=119)\end{array}$} & Até 20 anos & $25(41,7)$ & $35(58,3)$ & 1,042 & 0,91 \\
\hline & Acima de 20 anos & $24(40,7)$ & $35(59,3)$ & $0,50-2,16$ & \\
\hline \multirow{2}{*}{$\begin{array}{l}\text { Uso anterior de PI } \\
(\mathrm{N}=138)\end{array}$} & Sim & $34(47,9)$ & $37(52,1)$ & 1,646 & 0,15 \\
\hline & Não & $24(35,8)$ & $43(64,2)$ & $0,86-3,26$ & \\
\hline \multirow{2}{*}{$\begin{array}{l}\text { Tipo anterior de PI } \\
(\mathrm{N}=71)\end{array}$} & PTC & $31(52,5)$ & $28(47,5)$ & 3,321 & 0,115 \\
\hline & PPR & $3(25)$ & $9(75)$ & $0,82-13,51$ & \\
\hline \multirow{2}{*}{$\begin{array}{l}{ }^{* *} \text { Tempo de uso da PI } \\
\text { anterior }(\mathrm{N}=71)\end{array}$} & Até 1,5 anos & $0(0)$ & $2(100)$ & & \\
\hline & Acima de 1,5 anos & $34(49,3)$ & $35(50,7)$ & & \\
\hline \multirow{2}{*}{$\begin{array}{l}\text { Qualidade técnica PTC } \\
\text { superior }(\mathrm{N}=138)\end{array}$} & Satisfatória & $30(38)$ & $49(62)$ & 0,678 & 0,264 \\
\hline & Insatisfatória & $28(47,5)$ & $31(52,5)$ & $0,34-1,34$ & \\
\hline \multirow{2}{*}{$\begin{array}{l}\text { Qualidade técnica PTC } \\
\text { inferior }^{*}(\mathrm{~N}=65)\end{array}$} & Satisfatória & $2(33,3)$ & $4(66,7)$ & 0,422 & 0,413 \\
\hline & Insatisfatória & $32(54,2)$ & $27(45,8)$ & $0,07-2,48$ & \\
\hline \multirow{2}{*}{$\begin{array}{l}\text { Dentes funcionais } \\
(\mathrm{N}=138)\end{array}$} & Presença & $16(28,1)$ & $41(71,9)$ & 0,362 & 0,005 \\
\hline & Ausência & $42(51,9)$ & $39(48,1)$ & $0,18-0,75$ & \\
\hline \multirow{2}{*}{$\begin{array}{l}\text { Lesão pela prótese } \\
(\mathrm{N}=138)\end{array}$} & Presença & $17(48,6)$ & $18(51,4)$ & 1,428 & 0,364 \\
\hline & Ausência & $41(39,8)$ & $62(60,2)$ & $0,66-3,09$ & \\
\hline \multirow{2}{*}{$\begin{array}{l}\text { Sangramento gengival } \\
(\mathrm{N}=56)\end{array}$} & Presença & $6(31,6)$ & $13(68,4)$ & 1,436 & 0,56 \\
\hline & Ausência & $9(24,3)$ & $28(75,7)$ & $0,42-4,89$ & \\
\hline \multirow{2}{*}{$\begin{array}{l}\text { Cálculo dentário } \\
(\mathrm{N}=56)\end{array}$} & Presença & $10(33,3)$ & $20(66,7)$ & 2,100 & 0,365 \\
\hline & Ausência & $5(19,2)$ & $21(80,8)$ & $0,61-7,23$ & \\
\hline Cárie dentária* & Presença & $1(50)$ & $1(50)$ & 2,857 & 0,468 \\
\hline $\mathrm{N}=56$ & Ausência & $14(25,9)$ & $40(74,1)$ & $0,17-48,80$ & \\
\hline
\end{tabular}

PS: Prótese superior; PI: Prótese inferior; PTC: Prótese Total convencional; PPR: Prótese Parcial Removível. *Exato de Fisher. "* Testes estatísticos não se aplicam

tam às limitações das PTC. Essas inferências estão em consonância com a argumentação de que a maioria dos usuários de PTC está satisfeita com os benefícios desses dispositivos ${ }^{40}$, independente da qualidade técnica dessas próteses ${ }^{32}$.

Entretanto, outros estudos constataram associação entre indicadores subjetivos e clínicos, ou seja, verificaram que PTC com adaptação e retenção inadequadas tornavam seus usuários mais passíveis de relatarem dificuldades em seu dia-a-dia, após ajuste do efeito da idade, gênero e nível educacional ${ }^{40,41}$. Também objetivando comprovar essa associação, Shigli e Hebbal ${ }^{42}$ compararam a QVSB de pessoas reabilitadas com 
PTC tecnicamente satisfatórias antes e 1 mês após tal reabilitação. Esses autores constataram uma melhora significativa da QVSB $(\mathrm{p}=0,002)$ das pessoas, reforçando a hipótese de que haveria uma associação entre a qualidade técnica e a subjetividade. Vale salientar que esses estudos ${ }^{40-43}$ incluíram somente indivíduos desdentados totais bimaxilares para verificar a associação entre as características clínicas e as perceptivas das próteses dentárias.

Esse critério de inclusão não ocorreu nesta pesquisa. Assim, tal fato poderia justificar a ausência de associação entre qualidade técnica e dificuldades diárias. Os indivíduos desdentados, monomaxilares incluídos, tinham dentes funcionais, sobretudo na região anterior inferior, e essa presença reduziria a probabilidade de relato de dificuldades diárias decorrentes das PTC. Essa hipótese é reforçada por estudos que constataram associação entre o número de dentes remanescentes e QVSB ${ }^{42,44,45}$. Os autores desses estudos ainda colocam que o número de dentes remanescentes seria mais importante que o tipo de prótese para explicar a QVSB. Nesse mesmo sentido, Bonan et al. ${ }^{46}$ não encontraram associação entre percepção de QV (GOHAI) e a presença ou a ausência de próteses ou sua condição e qualidade, bem como com a condição de saúde bucal do indivíduo.

Partindo para uma avaliação mais abrangente dos resultados, verificamos que, em geral, os CEO cumpriram sua função de assegurar o direito à assistência odontológica de média complexidade ${ }^{47}$. Isto ocorreu, sobretudo, entre os adultos e os idosos de baixa renda que caracterizaram o perfil dos usuários desses serviços. De fato, essa população concentra a necessidade de próteses, já que $69 \%$ dos adultos necessitam de algum tipo de prótese e 38\% dos idosos necessitam de $\mathrm{PTC}^{14}$. Assim, a assistência está se concentrando na população mais necessitada conforme as metas do Brasil Sorridente ${ }^{48}$.

Deve-se absorver a premissa de que um serviço de alta qualidade deve ter o usuário satisfeito. E para tal medição devem-se utilizar os indicadores perceptivos. Estes, aliados aos clínicos, fornecem um panorama mais aproximado da real condição do indivíduo ${ }^{49,50}$, possibilitando uma melhor efetividade e eficiência das intervenções. Este fato foi verificado por Srisilapanan e Sheiham ${ }^{23}$ que obtiveram uma redução de 40 a $50,5 \%$ na necessidade de tratamento com próte- ses parciais ao utilizar o indicador OIDP associado ao clínico.

Deste modo, seria possível averiguar, por exemplo, se a reabilitação com PTC iria realmente possibilitar uma QVSB satisfatória ao indivíduo, evitando custos com reabilitações inadequadas. Os recursos poupados poderiam ser investidos pelo Governo Federal na inclusão de outros dispositivos protéticos, tais como implantes, no leque de serviços dos CEO que pudessem atender às necessidades reais dos indivíduos, melhorando sua QVSB ${ }^{38}$.

Atualmente, o acompanhamento das atividades dos CEO é meramente quantitativo, através dos dados de produtividade presentes no Sistema de Informação Ambulatorial do SUS (SIA/ SUS ${ }^{12}$. Estes dados determinam o repasse financeiro mensal do Ministério da Saúde para o $\mathrm{Mu}$ nicípio/Estado para custeio da confecção das PTC $^{45,46}$. Este foco eminentemente quantitativo pode prejudicar a qualidade dos serviços de saúde, configurando-se em "indústrias de produção em série”, na medida em que desconsidera o objeto de sua prática, o ser humano e sua subjetividade $^{47}$. Isso reforça a importância de planejar e avaliar os serviços desses Centros através de indicadores clínicos e perceptivos ${ }^{48,49}$.

As principais limitações deste estudo estão relacionadas às dificuldades inerentes às pesquisas de avaliação dos serviços, sobretudo à composição da amostra. A população sob estudo apresentava alta mobilidade o que dificultou a seleção. Os registros dos CEO estavam desatualizados e muitas pessoas haviam mudado de telefone ou de endereço.

\section{Conclusão}

Os CEO foram efetivos na reabilitação com PTC superiores, uma vez que as dificuldades encontradas estão dentro das limitações próprias a essa reabilitação. Contudo, a reabilitação mandibular necessita de uma reavaliação quanto ao seu custo-benefício, já que no total as PTC inferiores estavam bastante insatisfatórias.

Assim, é preciso instituir uma avaliação periódica desses Centros, agregando indicadores normativos e perceptivos, para obter um diagnóstico mais acurado das demandas coletivas e, então, alcançar a resolutividade e a racionalidade na alocação de recursos. 


\section{Colaboradores}

FCA Machado trabalhou na concepção, delineamento da pesquisa, análise e interpretação dos dados e redação do manuscrito. APS Costa e ALB Pontes trabalharam na concepção da pesquisa, coleta e análise dos dados. KC Lima e MAF Ferreira trabalharam na análise e interpretação dos dados e revisão crítica do artigo.

\section{Referências}

1. Castiel LD. Promoção de saúde e a sensibilidade epistemológica da categoria 'comunidade'. Rev Saude Publica 2004; 38(5):615-622.

2. Seidl EMF, Zannon CMLC. Qualidade de vida e saúde: aspectos conceituais e metodológicos. Cad Saude Publica 2004; 20(2):580-588.

3. Minayo MCS, Hartz ZMA, Buss PM. Qualidade de vida e saúde: um debate necessário. Cien Saude Colet 2000; 5(1):7-18.

4. Adulyanon $S$, Sheiham A. Oral Impacts on Daily Performances. In: Slade GD, editor. Measuring oral health and quality of life. Chapel Hill: School of Dentistry, University of North Carolina; 1997. p.152-160.

5. Gomes AS, Abegg C. O impacto odontológico no desempenho diário dos trabalhadores do Departamento Municipal de Limpeza Urbana de Porto Alegre, Rio Grande do Sul, Brasil. Cad Saude Publica 2007; 23(7):1707-1714.

6. Goes PSA. The prevalenceand impact of dental pain in Brazilian schoolchildren and their families [dissertation]. London: University College London; 2001.

7. Cortes MI, Marcenes W, Sheiham A. Impact of traumatic injuries to the permanent teeth on theoral health-related quality of life in 12-14-year-old children. Community Dent Oral Epidemiol 2002; 30(3):193-198.

8. Michel-Crosato E, Biazevic MG, Crosato E. Relationship between dental fluorosis and quality of life: a population based study. Braz Oral Res 2005; 19(2):150-155.

9. Botazzo C. Sobre a bucalidade: notas para a pesquisa e contribuição ao debate. Cien Saude Colet 2006; 11(1):7-17.

10. Barros RS, Botazzo C. Subjetividade e clínica na atenção básica. narrativas, histórias de vida e realidade social. Cien Saude Colet 2011; 16(11):4337-4348.

11. Vilella W. De saúde bucal, saúde mental, saúde sexual: o reiterado retorno à clínica. Cien Saude Colet 2006; 11(1):18-43.

12. Brasil. Ministério da Saúde (MS). Centros de Especialidades e Laboratórios de Prótese dentária. [página na Internet]. [acessado $2011 \mathrm{fev} \mathrm{16].} \mathrm{Disponí-}$ vel em: http://portal.saude.gov.br/portal/saude/ visualizar_texto.cfm?idtxt $=23646 \&$ janela $=1$

13. Brasil, Ministério da Saúde (MS). Secretaria de Atenção à saúde. Departamento de Atenção Básica. Projeto SB Brasil 2003: condições de saúde bucal da população brasileira 2002-2003: resultados principais. Brasília: MS; 2004.

14. Brasil. Ministério da Saúde (MS). Secretaria de Vigilância em Saúde. Secretaria de Assistência à Saúde. Departamento de Atenção Básica. Coordenação Nacional de Saúde Bucal. Pesquisa Nacional de Saúde Bucal-2010: Nota para a Imprensa. [acessado 2011 abr 2005]. Disponível em: http://www.idisa.org.br/ img/File/SAUDE\%20BUCAL-NotaParaImprensa28dez2010\%20\%282\%29.pdf 
15. Redford M, Drury T, Kingman A, Brown L. Denture use and the technical quality of dental prostheses among persons 18-74 years of age: United States, 1988-1991. J Dent Res 1996; 75(No esp.):714725.

16. Brasil. Lei no 8142 , de 28 de Dezembro de 1990. Dispõe sobre a participação da comunidade na gestão do Sistema Único de Saúde (SUS) e sobre as transferências intergovernamentais de recursos financeiros na área da saúde e dá outras providências. Diário Oficial da União 1990; 31 dez.

17. Brasil. Ministério da Saúde (MS). Cidades atendidas. [página na Internet]. [acessado 2009 mar 20]. Disponível em: http://dtr2004.saude.gov.br/dab/ cnsb/cidades_atendidas.php

18. Rio Grande do Norte. Conselho Regional de Odontologia do Rio Grande do Note. Canguaretama e Macaíba vão representar o RN no Prêmio Brasil Sorridente 2010. [página na Internet]. [acessado 2011 out 19]. Disponível em: http://www.crorn.org.br/ noticias/ver/485

19. Sheiham A, Tsakos G. Avaliando Necessidades Através de Abordagem Sócio - Odontológica. In: Pinto VG. Saúde Bucal Coletiva. São Paulo: Santos; 2008. p. $287-316$

20. Brasil. Ministério da Saúde (MS). Secretaria de Políticas de Saúde. Departamento de Atenção Básica. Coordenação Nacional de Saúde Bucal. Projeto $S B$ Brasil 2010: Pesquisa Nacional de Saúde Bucal Manual da Equipe de Campo. Brasília: MS; 2009.

21. Gherunpong S, Tsakos G, Sheiham A. The prevalence and severity of oral impacts on daily performances in Thai primary school children. Health qual. life outcomes 2004; 2:57.

22. Gladis MM, Gosch EA, Dishuk NM, Cris- Cristoph P. Quality of life: expanding the scope of clinical significance. J Consult Clin Psychol 1999; 67(3):320331.

23. Srisilapanan P, Sheiham A. Assessing the difference between sociodental and normative approaches to assessing prosthetic dental treatment needs in dentate older people. Gerodontology 2001; 18(1):25-34.

24. Berg E. Acceptance of full dentures. Int Dent J 1993; 43(Supl. 1):299-306.

25. van Waas MA. Determinants of dissatisfaction with dentures: a multiple regression analysis. J Prost Dent 1990; 64(5):569-572.

26. van Waas MA. The influence of psychologic factors on patient satisfaction with complete dentures. $J$ Prost Dent 1990; 63(5):545-548.

27. Kapur KK. Management of the edentulous elderly patient. Gerodontics 1987; 3(1):51-54.

28. Steele JG. How do age and tooth loss affect oral health impacts and quality of life? A study comparing two national samples. Community Dent Oral Epidemiol 2004; 32(2):104-114.
29. Akifusa S, Soh I, Ansai T, Hamasaki T, Takata Y, Yoshida A, Fukuhara M, Sonoki K, Takehara T. Relationship of number of remaining teeth to health-related quality of life in community-dwelling elderly. Gerodontology 2005; 22(2):91-97.

30. Astrøm AN, Haugejorden O, Skaret E, Trovik TA, Klock KS. Oral impacts on daily performance in Norwegian adults: the influence of age, number of missing teeth, and socio-demographic factors. Eur J Oral Sci 2006; 114(2):115-121.

31. Heydecke G, Thomasona MJ, Lunda JP, Feine JS. The impact of conventional and implant supported prostheses on social and sexual activities in edentulous adults Results from a randomized trial 2 months after treatment. J Dent 2005; 33(8):649-657.

32. Critchlow SB, Ellis JS. Prognostic indicators for conventional complete denture therapy: a review of the litterature. J Dent 2010; 38(1):2-9.

33. Reisine ST, Bailit HL. Clinical oral health status and adult perceptions of oral health. Soc Sci Med 1980; 14A(6):597-605.

34. Cushing AM, Sheiham A, Maizels J. Developing social-dental indicators: the social impact of dental disease. Community Dent Health 1986; 3(1):3-17.

35. Wilson B, Clearly PD. Linking clinical variables with health related quality of life: conceptual model of patient outcomes. JAM 1995; 273(1):59-65.

36. Pucca Júnior GA. A saúde bucal do idoso? Aspectos demográficos e epidemiológicos. 2000. [página na Internet]. [acessado $2010 \mathrm{fev} \mathrm{16].} \mathrm{Disponível} \mathrm{em:}$ http://www.odontologia.com.br/artigos.asp?id= 81\&idesp $=19$ \&ler $=5$

37. Rosa AG, Fernandez RAC, Pinto VG, Ramos LR. Condições de saúde bucal em pessoas de 60 anos ou mais no Município de São Paulo (Brasil). Rev Saude Publica 1992; 26(3):155-160.

38. Carlsson GE, Omar R. The future of complete dentures in oral rehabilitation: A critical review. J Oral Rehabil 2010; 37(2):143-156.

39. Bae KH, Kim C, Paik DI, Kim JB. A comparison of oral health related quality of life between complete and partial removable denture-wearing older adults in Korea. J Oral Rehabil 2006; 33(5):317-322.

40. Bagewitz IC, Söderfeldt B, Palmqvist S, Nilner K. Oral Prostheses and Oral Health-Related Quality of Life: A Survey Study of an Adult Swedish Population. Int J Prosthodont 2007; 20(2):132-142.

41. Tsakos G, Mrcenes W, Sheiham A. The relationship between clinical dental status and oral impacts in a elderly population. Oral Health Prev Dent 2004; 2(3):211-220

42. Shigli K, Hebbal M. Assessment of changes in oral health related quality of life among patients with complete denture before and 1 month post- insertion using Geriatric Oral Health Assessment Index. Gerodontology 2010; 27(3):167-173. 
43. Tsakos G, Steele JG, Marcenes W, Walls AWG, Sheiham A. Clinical correlates of oral health-related quality of life: evidence from a national sample of Britsh older people. Eur J Oral Sci 2006; 114(5): 391-395.

44. Elias AC, Sheiham A. The relationship between satisfaction with mouth and number and position of teeth. J Oral Rehabil 1998; 25(9):649-661.

45. Yoshida Y, Hatanaka Y, Imaki M, Ogawa Y, Miyatani S, Tanada S. Epidemiological study on improving the QOL and oral conditions of the aged - Part 1: The relationship between the status of tooth preservation and QOL. J Physiol Anthropol Appl Human Sci 2001; 20(6):363-368.

46. Bonan PRF, Borges SP, Haikal DS, Silveira MF, Martelli-Júnior H.Condições bucais e de reabilitação insatisfatórias dissociadas da percepção de qualidade de vida em idosos institucionalizados e não-institucionalizados. Rev odonto ciênc 2008; 23(2):115119.

47. Saliba NA, Moimaz SAS, Fadel CB, Bino LS. Saúde Bucal no Brasil: uma nova política de enfrentamento para a realidade nacional. Rev Odontol Bras Central 2010; 18(48):62-66.

48. Brasil. Ministério da Saúde (MS). Secretaria de Atenção à Saúde. Departamento de Atenção básica, Coordenação Nacional de Saúde bucal. Política Nacional de Saúde Bucal. Brasília: MS; 2004

49. Silva SRC. Autopercepção das condições bucais em pessoas com 60 anos e mais [tese]. São Paulo: Universidade de São Paulo; 1999.

50. Biazevic MG. Indicadores subjetivos em saúde bucal: uma revisão sistemática. 2001. Dissertação [dissertação]. São Paulo: Universidade de São Paulo; 2001.

Artigo apresentado em 29/03/2012

Aprovado em 21/04/2012

Versão final apresentada em 07/05/2012 